\title{
A Polar Representation for Complex Interval Numbers
}

\author{
Aarão Lyra \\ Information Systems and Engineering of Computation Courses \\ Potiguar University, UnP \\ Natal, RN, Brazil \\ aarao@unp.br
}

\author{
Benjamín René Callejas Bedregal \\ Department of Informatic and Applied Mathematics \\ Federal University of Rio Grande do Norte \\ Natal, RN, Brazil. \\ bedregal@dimap.ufrn.br
}

\begin{abstract}
The present work defines the basic elements for the introduction to the Study of Complex variables under the mathematical interval context with the goal of using it as a foundation for the understanding of pure mathematical problems, associating the mathematical interval to support the results. The present article contributes to the complex interval theory taking into consideration Euler's Identity and redefining the polar representation of interval complex numbers. In engineering, the present article could be used as a subsidy for many applications where complex variable theory is applicable and requires accurate results.
\end{abstract}

Index Terms-Complex Interval, Complex Interval Numbers, Complex Interval Variable

\section{INTRODUCTION}

In pure and applied mathematics there are problems involving continuous sets as real and complex numbers. This knowledge can be employed in engineering sciences, such as numerical analysis, dynamic systems, fazorial analysis [7], computational geometry [21], signals processing and digital images [6], [12] as well as the theory of optimization among others. However, in most cases there is no numerical error analysis, thus taking for granted the uncertainty and insecurity of the results. Thus there is a necessity for an approach to solve continuous problems using mathematical intervals.

Under the topic of real numbers, several approaches on intervals are well known [13], [14], [15] [1], [17] and [10] however, there is little work done on or few references are available on complex numbers and this field needs to be enriched.

In this work, there are definitions of some basic elements on complex interval theory, which is a mathematical theory on interval of complex numbers that serve as a foundation for problems on mathematical applications but at the same time associating it with mathematical interval to offer reliability to the results that are being introduced.

\author{
Adrião Duarte Dória Neto \\ Department of Computation and Automation Engineering \\ Federal University of Rio Grande do Norte \\ Natal, RN, Brazil \\ adriao@dca.ufrn.br
}

\author{
Roque Mendes Prado Trindade \\ Department of Thecnologics an Exacts Sciences \\ State University of Southwest Bahia - (UESB) \\ Vit. Conquista, Ba, Brazil. \\ roquetrindade@uesb.edu.br
}

The authors believe that the present work contributes to the use of complex data number type that needs precision and accuracy on complex numerical data treatment as well as an addition to the mathematical literature. Definitions are suggested in this article, under the understanding of complex numbers and introducing Euler's identity solutions for the interval case and defining a polar representation. For this, it was necessary to evaluate the current information available on the main definitions of interval complex number and their influence on some of the jobs selected in this area [4] and [16].

This work also attenps to fill in a gap in mathematical modelling in the research area of robotics, sensors fusion, autonomous tracking, innacurate robot lacalization, such as in the work by Kieffer, Jaulin and Walter [9]. Jaulin uses interval analysis for planning free-collision paths for mobile robots [8]. Interval analysis was also used for vehicle localization to solve problems of telemetric imprecision data by Leveque in [11] and it is used as fundamentation of interval digital signal processing in [18].

\section{THe COMPLEX Numbers}

In the XV Century mathematicians like Cardano and Bombelli, among others, carried out studies on negative numbers. Two centuries later, Wesses, Argand and Gauss continued this work and they are being considered as the creators of the theory of complex numbers, which became an important tool in engineering.

\section{A. Basic Definitions}

\section{Definition 1 (Complex numbers):}

Let $a, b \in \mathbb{R}$ and $z=a+b i$, where $i=\sqrt{-1}$, then, $z$ is called a complex number.

The set of all complex numbers is called complex plan and it is denoted by $\mathbb{C}$. 
This set provides arithmetical operations such as addition, subtraction, multiplication, and division.

Geometrically, a complex number is seen as a point in the complex plan, as viewed in figure 1 .

Fig. 1. Cartesian representation of a complex number

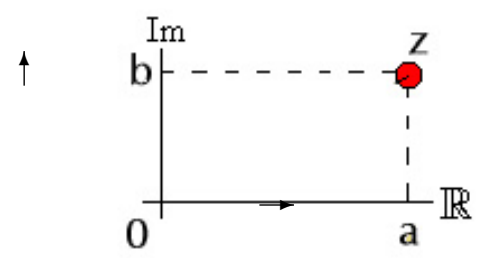

Definition 2 (Equality between complex numbers):

Let $x, y \in \mathbb{C}$ where $x=a+b i$ and $y=c+d i . x=y$ if $a=c$ and $b=d$.

Definition 3 (Conjugation of a complex number):

Let $z=a+b i \in \mathbb{C}$. $\bar{z}=a-b i$ is the conjugation of $z$.

Definition 4 (Addition, subtraction and multiplication on $\mathbb{C}$ ):

Let $x, y \in \mathbb{C}$ where $x=a+b i$ and $y=c+d i$. The operations of addition, subtraction and multiplication in $\mathbb{C}$ are defined through the intervals of basic arithmetical operations:

- $x+y=(a+b i)+(c+d i)=(a+c)+(b+d) i$

- $x-y=(a+b i)-(c+d i)=(a-c)+(b-d) i$

- $x \times y=(a+b i) \times(c+d i)=(a c-b d)+(a d+b c) i$

Definition 5 (Inverse of the complex numbers):

Let $z=a+b i$, the inverse of $z$, denoted $z^{-1}$, is the complex number, so that $z \times z^{-1}=1$

Definition 6 (Division between complex numbers):

$x \div y=x \times y^{-1}$ for $y \neq 0+0 i$

Definition 7 (Module of a complex number):

Let $z \in \mathbb{C}$ where $z=a+b i$. Then the real number

$$
|z|=\sqrt{a^{2}+b^{2}}
$$

is called module of $\mathrm{z}$.

Definition 8 (Distance between complex numbers):

Let $x=a+b i$ and $y=c+d i \in \mathbb{C}$. The function $d_{c}: \mathbb{C} \times \mathbb{C} \rightarrow \mathbb{R}$,defined by $d_{c}(x, y)=\sqrt{(a-c)^{2}+(d-b)^{2}}$, is called the distance in between $x, y$.

Theorem 2.1:

$d_{c}$ is a metric.

Proof: This metric $d_{c}$ is congruent with euclidian metric on the space $\mathbb{R}^{2}$.

\section{B. Order in complex numbers}

It is known that the complex numbers are not totally ordered, but different partially ordered complex numbers exist. In some of the problems in physics, a partial order based on the understanding of module is used, where the distance of the vector from a point of origin will be analyzed.

Definition 9 (Partial order in a set of complex numbers using module):

Let $x, y \in \mathbb{C}$. $x$ is less than or equal to $y$, denoted by $x \preceq y$, if $|x| \leq|y|$.

In this order the complex number $z_{1}$ is less than the complex number $z_{2}$ if the point $z_{2}$ is away from the origin than the point $z_{1}$. It is well known that the real numbers set is immersed in the complex numbers set, but the usual immersion $(f(r)=r+$ $0 i$ ) and this order on the complex numbers does not preserve the usual real order, for example, $-2 \leq 1$ in real numbers, but clearly $-2+0 i \npreceq 1+0 i$.

Another natural partial order on the set of a complex number is a constructed one, by analyzing the geometrical position of the complex numbers.

Definition 10 (Natural partial order in a set of complex numbers):

Let $x, y \in \mathbb{C}$. $x$ is less or equal to $y$, denoted by $x \leq y$, if exists $n \in \mathbb{C}^{+}=\{a+b i \mid a, b \geq O\}$ so that $x+n=y$.

Note that, trivially, this order preserves the usual real order, i.e. if $r \leq s$ then $r+0 i \leq s+0 i$.

\section{Proposition 2.2:}

Let $x, y \in \mathbb{C}$, where $x=a+b i$ and $y=c+d i$. $x \leq y$, if and only if, $a \leq c$ and $b \leq d$.

\section{Proof:}

$\Rightarrow$ Let $x=a+b i, y=c+d i \in \mathbb{C}$. If $x \leq y$ then $\exists n=$ $e+f i \in \mathbb{C}^{+}$so that, $x+n=y$.

So $(a+b i)+(e+f i)=c+d i a+b i+e+f i=c+d i$ $a+e+b i+f i=c+d i(a+e)+(b+f) i=c+d i$. Therefore, $c=a+e$ and $d=b+f$ if $e, f \geq 0$, then $a \leq c$ and $b \leq d$.

$\Leftarrow$ Let $a, b, c, d \in \mathbb{R}$. If $a \leq c$ and $b \leq d$ then $\exists e, f \in \mathbb{R}^{+}$ so that, $c=a+e$ and $d=b+f$.

Let $x=a+b i, y=c+d i$ and $n=e+f i$.

$$
y=c+d i
$$$$
=(a+e)+(b+f) i
$$

Thus $\quad=a+e+b i+f i$

$$
=a+b i+e+f i
$$

$$
=x+n \text {. }
$$

Consequently, $\quad x \leq y$.

In this work the natural partial order on the set of complex numbers will be only used for the construction of the complex interval numbers, as can be seen in figures 3 and 4, and for the arithmetical operations. In physical applications the partial order in a set of complex numbers using module will be used.

\section{Polar representation of complex numbers}

We may recall that each point $P$ of the plane and hence each complex number $z$ is uniquely determined by two polar coordinates $\rho$ and $\alpha$, where $\rho$ is the length (non-negative) of the segment $\overrightarrow{o p}$, which joins the point $P$ to the origin and $\alpha$ is the angle formed between the $x$ axis and this segment, which 
is called the argument or amplitude of $P$. We can see it in figure 2 .

Fig. 2. Graph representation of polar form of a complex number

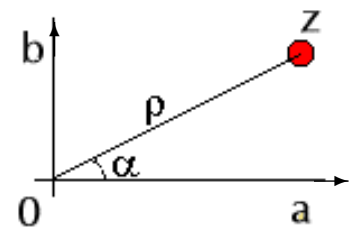

Considering the trigonometric relations from polar representation, as can be seen in figure 2, the polar coordinates $(\rho, \alpha)$ of the point $z=a+b i$ can be determined as follows: $a=\rho \cos (\alpha)$ and $b=\rho \sin (\alpha)$ Thus,

$$
\begin{aligned}
z & =a+b i \\
& =\rho \cos (\alpha)+\rho \sin (\alpha) i \\
& =\rho[\cos (\alpha)+i \sin (\alpha)] .
\end{aligned}
$$

Conversely, the polar coordinates can be obtained from their Cartesian coordinates as follows: $\rho=|z|=\sqrt{a^{2}+b^{2}}$ and $\alpha=\arctan \left(\frac{b}{a}\right)$.

Theorem 2.3: Euler's identity

$e^{i \alpha}=\cos (\alpha)+i \sin (\alpha)$

Proof: The proof of this theorem can be seen in [20].

\section{COMPLEX INTERVAL NUMBers}

The complex number has the property of representation and manipulation of two variables as a single quantity. This is quite natural with the Fourier analysis, where the field of frequency is composed by two signals, the real and imaginary part. In some way the complex number simplifies the equations used in Digital Signal Processing (DSP) and provides techniques which could be difficult or impossible only with real numbers. This work proposes an interval version for complex numbers for use in signal processing, where we intend to solve the problem mentioned by Boche [4] i.e. the incompatibilization between the rectangular form and the polar form of interval complex numbers which can be seen in more recent work as [3], [5] and [2]. To do so, we describe complex interval numbers, their rectangular form, their polar form and their properties and we present a version of Euler's identity for interval complex numbers.

The complex intervals are a generalization of the real intervals and it could be seen that any real interval can be seen as a real complex interval. However, some of the definitions and their implications that will contribute to the different fields of engineering in their future applications are presented here.

Definition 11 (Complex interval):
Let $A, B \in \mathbb{I}$. $Z=A+B i$, where $i=\sqrt{-1}$ is called an interval complex number. The set formed by all the interval complex numbers is denoted by $\mathbb{I} \mathbb{C}$.

\section{Observation 3.1:}

Geometrically, an interval complex number is seen as a closed rectangular region in the complex plane, whose sides are parallel to the coordinates. It can be constructed through the intersection of the regions shown in figure 3 .

$$
\begin{aligned}
\text { Let } & A=\left[a_{1}, a_{2}\right], B=\left[b_{1}, b_{2}\right] \in \mathbb{I} \text {. Note that, } \\
Z & =A+B i \\
& =\left[a_{1}, a_{2}\right]+\left[b_{1}, b_{2}\right] i \\
& =\left[a_{1}, a_{2}\right]+\left[b_{1} i, b_{2} i\right] \\
& =\left[a_{1}+b_{1} i, a_{2}+b_{2} i\right] \\
& =\left\{c \in \mathbb{C} \mid a_{1}+b_{1} i \leq c \leq a_{2}+b_{2} i\right\} .
\end{aligned}
$$

Thus, an interval complex number can be identified with an interval of complex numbers. This formation can be better seen in figures 4,5 and 6.

From definition 11, one could see that the complex intervals generalize the real intervals; moreover, every complex number is a complex interval.

a If $A \in \mathbb{R}$, it could be represented as $A+[0,0] i$, therefore $A \in \mathbb{I} \mathbb{C}$;

b If $z=a+b i \in \mathbb{C}$, then

$$
\begin{aligned}
Z & =[a, a]+[b, b] i \\
& =[a+b i, a+b i] \\
& =[z, z] \\
& =\{c \in \mathbb{C} \mid z \leq c \leq z\} \\
& =\{z\}
\end{aligned}
$$

Thus, $z$ can be represented by the degenerate complex interval $Z=\{z\}$, therefore $z \in \mathbb{I C}$.

Note: In this work we consider

$$
A^{2}=\left[\max \left\{0, \min \left\{a_{1} a_{2}, a_{1}^{2}, a_{1}^{2}\right\}\right\} ; \max \left\{a_{1} a_{2}, a_{1}^{2}, a_{1}^{2}\right\}\right] .
$$

Definition 12 (Arithmetical operations between complex intervals):

Let $X=A+B i$ and $Y=C+D i \in \mathbb{I} \mathbb{C}$. The arithmetical operations addition, subtraction, multiplication and division in $\mathbb{I C}$ are defined through their respective real interval arithmetical operations:

- $X+Y=(A+C)+(B+D) i$

- $X-Y=(A-C)+(B-D) i$

- $X \times Y=(A C-B D)+(A D+B C) i$

- $X \div Y=\frac{A C-B D}{C^{2}+D^{2}}+\frac{B C-A D}{C^{2}+D^{2}} i$ for $0 \notin\left(C^{2}+D^{2}\right)$

\section{A. Order in Interval Complex Numbers}

Several orders have been defined about the real interval numbers ( Moore [14], Kulisch-Miranker [10], Moore's inclusion order [14] and information order by Scott [19] and Acióly [1]), and these sets can be extended to the interval complex numbers. In this article, we extended Kulisch-Miranker's order for the interval complex number as follows:

Definition 13 (Kulisch-Miranker's order for interval complex numbers): 
Fig. 3. Cartesian representation of the interval complex number $Z=A+B i$

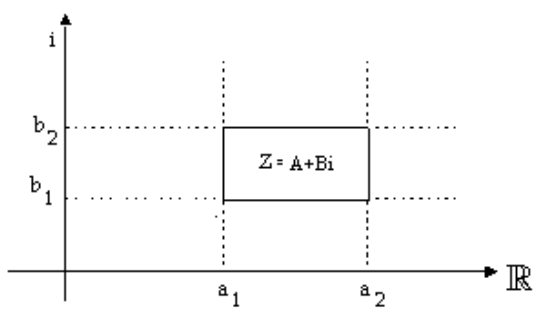

Fig. 4. The set $\uparrow A=\{c \in \mathbb{C} \mid A<c\}$

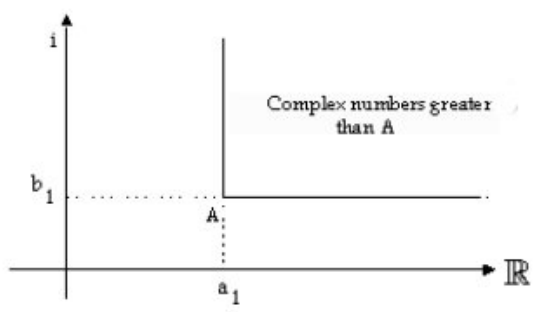

Let $X, Y \in \mathbb{I} C$. $X$ is less or equal to $Y$, denoted by $X \leq Y$, if exists

$$
N \in \mathbb{I} \mathbb{C}^{+}=\left\{A+B i \mid[0,0] \leq_{K M} A, B\right\}
$$

${ }^{1}$ so that $X+N=Y$.

Proposition 3.1:

Let $X, Y \in \mathbb{I} \mathbb{C}$, where $X=A+B i$ and $Y=$ $C+D i . X \leq Y$, if and only if, $A \leq_{K M} C$ and $B \leq_{K M} D$.

Proof:

$\Rightarrow$ Let $X=A+B i$ and $Y=C+D i \in \mathbb{I} \mathbb{C}$ so that $X \leq Y$. Then, by definition, there exists $N=E+F i \in \mathbb{I} \mathbb{C}^{+}$so that, $X+N=Y$.

So $(A+B i)+(E+F i)=C+D i A+B i+E+F i=C+D i$ $A+E+B i+F i=C+D i(A+E)+(B+F) i=C+D i$. Therefore $C=A+E$ and $D=B+F$. Since $[0,0] \leq_{K M} E, F$ then $A \leq_{K M} C$ and $B \leq_{K M} D$.

$\Leftarrow$ Let $A, B, C, D \in \mathbb{R}$ where $A \leq_{K M} C$ and $B \leq_{K M} D$. So, $\exists E, F \in \mathbb{I R}^{+}$so that, $C=A+E$ and $D=B+F$. Thus making, $X=A+B i, Y=C+D i$ and $N=E+F i$ we $Y=C+D i$

have that: $\quad=A+E+B i+F i$

Therefore, $X \leq Y$.

$$
\begin{aligned}
& =A+B i+E+F i \\
& =X+N .
\end{aligned}
$$

Definition 14 (Equality between interval complex numbers): Let $X=A+B i$ and $Y=C+D i$ where $X, Y \in \mathbb{I} \mathbb{C}$. Then $X=Y$ if $A=C$ and $B=D$

Definition 15 (Comparability):

Let $X, Y \in \mathbb{I C}$. $X$ and $Y$ are comparable if $X \leq Y$ or $Y \leq X$.

\section{B. Algebraic Properties of the Interval Complex Numbers}

In this subsection, some of the main algebraic properties about the complex intervals are defined: Let $X=[a, b]+[c, d] i, \quad Y=[e, f]+[g, h] i$ and $Z=[x, y]+[r, s] i$ where $X, Y, Z \in \mathbb{I} \mathbb{C}$.

- Commutativity of the Addition Operation: $X+Y=Y+X$

$$
\begin{aligned}
& \text { Proof: } X+Y=([a, b]+[e, f])+([c, d]+[g, h]) i \\
& =[a+e, b+f]+[c+g, d+h] i \\
& =[e+a, f+b]+[g+c, h+d] i \\
& =Y+X
\end{aligned}
$$

- Commutativity of the Multiplication Operation: $X \times Y=Y \times X$

${ }^{1}[r, s] \leq_{K M}[t, u]$, if only if, $r \leq t$ and $s \leq u$. 


\footnotetext{
Proof:

$X \times Y=$

$=([a, b] \times[e, f])-([c, d] \times[g, h])+$

$([a, b] \times[g, h])+([c, d] \times[e, f]) i$

$=([\min \{a \times e, a \times f, b \times e, b \times f\}+$

$\max \{a \times e, a \times f, b \times e, b \times f\}$

$-[\min \{c \times g, c \times h, d \times g, d \times h$, $\max \{c \times g, c \times h, d \times g, d \times h\}])$

$+([\min \{a \times g, a \times h, b \times g, b \times h\}$,

$\max \{a \times g, a \times h, b \times g, b \times h\}]$

$+\min \{c \times e, c \times f, d \times e, d \times f\}$,

$\max \{c \times e, c \times f, d \times e, d \times f\}]) i$

$=([\min \{e \times a, f \times a, e \times b, f \times b\}+$

$\max \{e \times a, f \times a, e \times b, f \times b\}$

$-[\min \{g \times c, h \times c, g \times d, h \times d\}$,

$\max \{g \times c, h \times c, g \times d, h \times d\}])+$

$([\min \{g \times a, h \times a, g \times b, h \times b\}$,

$\max \{g \times a, h \times a, g \times b, h \times b\}]$

$+\min \{e \times c, f \times c, e \times d, f \times d\}$,

$\max \{e \times c, f \times c, e \times d, f \times d\}]) i$

$=([\min \{a \times e, a \times f, b \times e, b \times f\}+$

$\max \{a \times e, a \times f, b \times e, b \times f\}$

$=([e, f] \times[a, b])-([g, h] \times[c, d])+$

$([g, h] \times[a, b])+([e, f] \times)[c, d] i$

$=Y \times X$
}

$$
\begin{aligned}
& \text { - Associativity of the Addition Operation: } \\
& X+(Y+Z)=(X+Y)+Z \\
& \text { Proof: } \\
& \quad X+(Y+Z) \\
& =[a, b]+[c, d] i+([e, f]+[g, h] i+[x, y]+[r, s] i) \\
& =[a, b]+[c, d] i+[e+x, f+y]+[g+r, h+s] i \\
& =[a+e+x, b+f+y]+[c+g+r, d+h+s] i \\
& =([a+e, b+f]+[c+g, d+h] i)+[x, y]+[r, s] i \\
& =(X+Y)+Z
\end{aligned}
$$

Other demonstrations can be seen in Oliveira [16]:

- Neutral Element of the Addition:

$$
\exists \quad 0=[0,0]+[0,0] i \in \mathbb{I} \mathbb{C}
$$

so that $X+0=0+X=X$

- Neutral Element of the Multiplication:

$$
\exists 1=[1,1]+[0,0] i \in \mathbb{I} \mathbb{C}
$$

so that $X \times 1=1 \times X=X$

- Sub-Distributive Property:

$$
X \times(Y+Z) \subseteq(X \times Y)+(X \times Z)
$$

C. Classical Polar Representation of Interval Complex Numbers

Definition 16 (Moore's interval complex distance):
Let $d_{M c}: \mathbb{I C} \times \mathbb{I C} \rightarrow \mathbb{R}$ the function is defined by:

$$
d_{M c}(A+B i, C+D i)=\operatorname{dist}(A, C)+\operatorname{dist}(B, D),
$$

where $\operatorname{dist}([r, s],[t, u])=\max \{|r-t|,|s-u|\}^{2} . d_{M c}$ is called Moore's complex distance.

Example 1:

Let $X=[2,5]+[2,3] i$ and $Y=[-2,-1]+[-1,1] i$ then $d_{M c}(X, Y)=$

$=\operatorname{dist}([2,5],[-2,-1])+\operatorname{dist}([2,3],[-1,-1])$

$=\operatorname{Max}(|2-(-2)|,|5-(-1)|)+\operatorname{Max}(|2-(-1)|,|3-(-1)|)$

$=6+4=10$

\section{Theorem 3.2:}

$d_{M c}$ is a metric

Proof: Let $X=A+B i, Y=C+D i, Z=F+E i \in \mathbb{I} \mathbb{C}$. Then

$d_{M c}(X, X)=$

$=\operatorname{dist}(A, A)+\operatorname{dist}(B, B)$

- $\max \{|\underline{A}-\underline{A}|,|\bar{A}-\bar{A}|\}+\max \{|\underline{B}-\underline{B}|,|\bar{B}-\bar{B}|\}$

$0+0$

$=0$

$d_{M c}(X, Y)=$

$=\operatorname{dist}(A, C)+\operatorname{dist}(B, D)$

- $=\operatorname{dist}(C, A)+\operatorname{dist}(D, B)$

$=d_{M c}(Y, X)$

- It has to be proved that

$$
\begin{gathered}
d_{M c}(X, Z) \leq d_{M c}(X, Y)+d_{M c}(Y, Z) \\
d_{M c}(X, Y)+d_{M c}(Y, Z)= \\
=\operatorname{dist}(A, C)+\operatorname{dist}(B, D)+\operatorname{dist}(C, F)+\operatorname{dist}(D, E) \\
=\operatorname{dist}(A, C)+\operatorname{dist}(C, F)+\operatorname{dist}(B, D)+\operatorname{dist}(D, E)
\end{gathered}
$$

as $\operatorname{dist}(A, F)$ and $\operatorname{dist}(B, E)$ are metric, then: $\operatorname{dist}(A, F) \leq \operatorname{dist}(A, C)+\operatorname{dist}(C, F)$ and $\operatorname{dist}(B, E) \leq$ $\operatorname{dist}(B, D)+\operatorname{dist}(D, E)$ thus $\operatorname{dist}(A, F)+\operatorname{dist}(B, E) \leq$ $\operatorname{dist}(A, C)+\operatorname{dist}(C, F)+\operatorname{dist}(B, D)+\operatorname{dist}(D, E)$ but

$$
\begin{aligned}
& d_{M c}(X, Z) \\
& =\operatorname{dist}(A, F)+\operatorname{dist}(B, E) \\
& \leq \operatorname{dist}(A, C)+\operatorname{dist}(C, F)+\operatorname{dist}(B, D)+\operatorname{dist}(D, E) \\
& =\operatorname{dist}(A, C)+\operatorname{dist}(B, D)+\operatorname{dist}(C, F)+\operatorname{dist}(D, E) \\
& =d_{M c}(X, Y)+d_{M c}(Y, Z)
\end{aligned}
$$

thus $d_{M c}(X, Z) \leq d_{M c}(X, Y)+d_{M c}(Y, Z)$

Other notions of distance:

Definition 17 (The interval distance between two interval complex numbers):

Let $X=A+B i$ and $Y=C+D i$ so that $X$ and $Y$ are comparable. The interval distance between $X$ and $Y$, denoted by $d I(X, Y)$, is:

$$
d I(X, Y)=\sqrt{(C-A)^{2}+(D-B)^{2}} .
$$

\footnotetext{
${ }^{2}$ dist is known for the Moore's distance [14] and is a metric.
} 
The construction of this definition basically came from Euclidian geometry, which is shown below. Let $X=A+B i$ and $Y=C+D i$; where $A=\left[a_{1}, a_{2}\right], B=\left[b_{1}, b_{2}\right], C=\left[c_{1}, c_{2}\right]$ and $D=\left[d_{1}, d_{2}\right] \in \mathbb{I} \mathbb{R}$; comparable complex intervals. The interval distance between $X$ and $Y$ can be seen in figure 7:

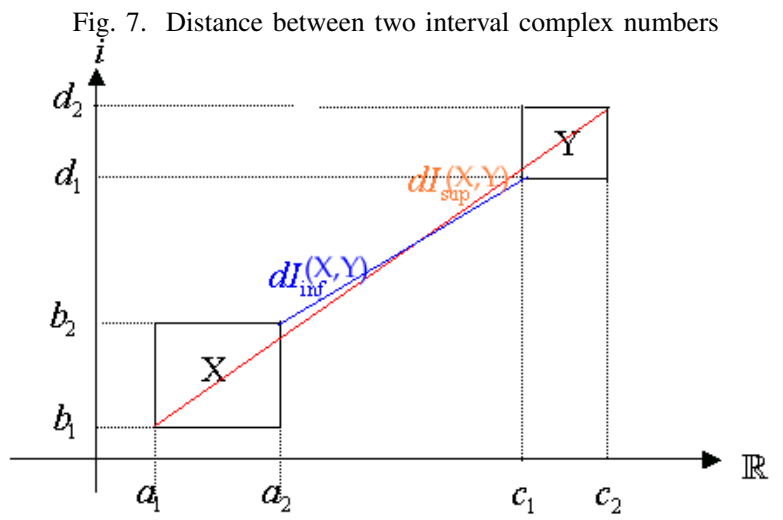

\section{Proposition 3.3:}

Let the interval complex numbers

$X=A+B i$ and $Y=C+D i$, where $A=\left[a_{1}, a_{2}\right]$,

$B=\left[b_{1}, b_{2}\right], C=\left[c_{1}, c_{2}\right]$ and $D=\left[d_{1}, d_{2}\right] \in \mathbb{I} \mathbb{R}$, so that

$X \leq Y$.

Then $d I(X, Y)=\left[d I_{\text {inf }}(X, Y), d I_{\text {sup }}(X, Y)\right]$,where

$$
\begin{aligned}
& d I_{\text {inf }}(X, Y)=\sqrt{\left(c_{1}-a_{2}\right)^{2}+\left(d_{1}-b_{2}\right)^{2}} \\
& d I_{\text {sup }}(X, Y)=\sqrt{\left(c_{2}-a_{1}\right)^{2}+\left(d_{2}-b_{1}\right)^{2}}
\end{aligned}
$$

Proof: if $X \leq Y$ then $A \leq C$ and $B \leq D$. So, $d I(X, Y)$

$=\sqrt{(C-A)^{2}+(D-B)^{2}}$

$=\sqrt{\left(\left[c_{1}, c_{2}\right]-\left[a_{1}, a_{2}\right]\right)^{2}+\left(\left[d_{1}, d_{2}\right]-\left[b_{1}, b_{2}\right]\right)^{2}}$

$=\sqrt{\left[c_{1}-a_{2} ; c_{2}-a_{1}\right]^{2}+\left[d_{1}-b_{2} ; d_{2}-b_{1}\right]^{2}}$

$=\sqrt{\left[\left(c_{1}-a_{2}\right)^{2} ;\left(c_{2}-a_{1}\right)^{2}\right]+\left[\left(d_{1}-b_{2}\right)^{2} ;\left(d_{2}-b_{1}\right)^{2}\right]}$

because $X \leq_{K M} Y$

$=\sqrt{\left[\left(c_{1}-a_{2}\right)^{2}+\left(d_{1}-b_{2}\right)^{2} ;\left(c_{2}-a_{1}\right)^{2}+\left(d_{2}-b_{1}\right)^{2}\right]}$

$=\left[\sqrt{\left(c_{1}-a_{2}\right)^{2}+\left(d_{1}-b_{2}\right)^{2}} ; \sqrt{\left(c_{2}-a_{1}\right)^{2}+\left(d_{2}-b_{1}\right)^{2}}\right]$

$=\left[d I_{\text {inf }}(X, Y), d I_{\text {sup }}(X, Y)\right]$

Thus, $\left[d I_{\inf }(X, Y), d I_{\text {sup }}(X, Y)\right]$ is the interval where its elements represent the minimum and maximum distances, respectively.

\section{Proposition 3.4:}

Let the interval complex numbers $X=A+B i$ and $Y=C+D i$, where $A=\left[a_{1}, a_{2}\right], B=\left[b_{1}, b_{2}\right], C=\left[c_{1}, c_{2}\right]$ and $D=\left[d_{1}, d_{2}\right] \in \mathbb{I}$. If $X \leq Y$ then $d I(X, Y)=d I(Y, X)$.

Proof: $d I(Y, X)$

$=\sqrt{(A-C)^{2}+(B-D)^{2}}$

$=\sqrt{\left(\left[a_{1}, a_{2}\right]-\left[c_{1}, c_{2}\right]\right)^{2}+\left(\left[b_{1}, b_{2}\right]-\left[d_{1}, d_{2}\right]\right)^{2}}$

$=\sqrt{\left[a_{1}-c_{2}, a_{2}-c_{1}\right]^{2}+\left[b_{1}-d_{2}, b_{2}-d_{1}\right]^{2}}$

$=\sqrt{\left[\left(a_{2}-c_{1}\right)^{2},\left(a_{1}-c_{2}\right)^{2}\right]+\left[\left(b_{2}-d_{1}\right)^{2},\left(b_{1}-d_{2}\right)^{2}\right]}$,

because $X \leq Y$

$=\sqrt{\left[\left(c_{1}-a_{2}\right)^{2} ;\left(c_{2}-a_{1}\right)^{2}\right]+\left[\left(d_{1}-b_{2}\right)^{2} ;\left(d_{2}-b_{1}\right)^{2}\right]}$

$=\sqrt{\left[c_{1}-a_{2} ; c_{2}-a_{1}\right]^{2}+\left[d_{1}-b_{2} ; d_{2}-b_{1}\right]^{2}}$

$=\sqrt{\left(\left[c_{1}, c_{2}\right]-\left[a_{1}, a_{2}\right]\right)^{2}+\left(\left[d_{1}, d_{2}\right]-\left[b_{1}, b_{2}\right]\right)^{2}}$

$=\sqrt{(C-A)^{2}+(D-B)^{2}}$

$=d I(X, Y)$.

Corollary 3.5:

Let $X, Y \in \mathbb{I} \mathbb{C}$. If $X$ and $Y$ are comparable, then $d I(X, Y)=\left[d I_{\text {inf }}(X, Y), d I_{\text {sup }}(X, Y)\right]$.

Proof: Straightforward from propositions 3.3 and 3.4.

Proposition 3.6:

Let the interval complex number $X=A+B i$, where $A=\left[a_{1}, a_{2}\right]$ and $B=\left[b_{1}, b_{2}\right]$. Thus $d I(X, X) \supseteq[0,0]$.

$$
\begin{aligned}
& \text { Proof: } \\
& d I(X, X) \\
& =\sqrt{(A-A)^{2}+(B-B)^{2}} \\
& =\sqrt{\left(\left[a_{1}, a_{2}\right]-\left[a_{1}, a_{2}\right]\right)^{2}+\left(\left[b_{1}, b_{2}\right]-\left[b_{1}, b_{2}\right]\right)^{2}} \\
& =\sqrt{\left[a_{1}-a_{2}, a_{2}-a_{1}\right]^{2}+\left[b_{1}-b_{2}, b_{2}-b_{1}\right]^{2}} \\
& =\sqrt{\left[0,\left(a_{2}-a_{1}\right)^{2}\right]+\left[0,\left(b_{2}-b_{1}\right)^{2}\right]} \\
& \text { for } a_{1} \leq a_{2} \quad \text { and } \quad b_{1} \leq b_{2} \\
& =\sqrt{\left[0,\left(a_{2}-a_{1}\right)^{2}+\left(b_{2}-b_{1}\right)^{2}\right]} \\
& =\left[\sqrt{0}, \sqrt{\left(a_{2}-a_{1}\right)^{2}+\left(b_{2}-b_{1}\right)^{2}}\right] \\
& =\left[0, \sqrt{\left.\left.\left(a_{2}-a_{1}\right)^{2}+\left(b_{2}-b_{1}\right)^{2}\right]\right]}\right. \\
& \supseteq[0,0]
\end{aligned}
$$

\section{Proposition 3.7:}

Let the interval complex numbers $X=A+B i$, $Y=C+D i$ and $Z=E+F i$, where $A=\left[a_{1}, a_{2}\right]$, $B=\left[b_{1}, b_{2}\right], C=\left[c_{1}, c_{2}\right], D=\left[d_{1}, d_{2}\right], E=\left[e_{1}, e_{2}\right]$ and $F=\left[f_{1}, f_{2}\right]$. If $X$ and $Y, Y$ and $Z$ and $X$ and $Z$ are comparable, then $d I(X, Y)+d I(Y, Z) \geq d I(X, Z)$.

Proof: We know $\quad$ that $d I(X, Y)$
$\left[\sqrt{\left(c_{1}-a_{2}\right)^{2}+\left(d_{1}-b_{2}\right)^{2}} ; \sqrt{\left(c_{2}-a_{1}\right)^{2}+\left(d_{2}-b_{1}\right)^{2}}\right]$
$d I(Y, Z)=$
$\left[\sqrt{\left(e_{1}-c_{2}\right)^{2}+\left(f_{1}-d_{2}\right)^{2}} ; \sqrt{\left(e_{2}-c_{1}\right)^{2}+\left(f_{2}-d_{1}\right)^{2}}\right]$ and
$d I(X, Z)=$
$\left[\sqrt{\left(e_{1}-a_{2}\right)^{2}+\left(f_{1}-b_{2}\right)^{2}} ; \sqrt{\left(e_{2}-a_{1}\right)^{2}+\left(f_{2}-b_{1}\right)^{2}}\right]$
$d I(X, Y)+d I(Y, Z)=$
$=\left[\sqrt{\left(c_{1}-a_{2}\right)^{2}+\left(d_{1}-b_{2}\right)^{2}} ; \sqrt{\left(c_{2}-a_{1}\right)^{2}+\left(d_{2}-b_{1}\right)^{2}}\right]+$
$\left[\sqrt{\left(e_{1}-c_{2}\right)^{2}+\left(e_{2}-c_{1}\right)^{2}} ; \sqrt{\left(f_{1}-d_{2}\right)^{2}+\left(f_{2}-d_{1}\right)^{2}}\right]=$
$=\left[\sqrt{\left(c_{1}-a_{2}\right)^{2}+\left(d_{1}-b_{2}\right)^{2}}+\sqrt{\left(e_{1}-c_{2}\right)^{2}+\left(e_{2}-c_{1}\right)^{2}} ;\right.$
$\left.\sqrt{\left(c_{2}-a_{1}\right)^{2}+\left(d_{2}-b_{1}\right)^{2}}+\sqrt{\left(f_{1}-d_{2}\right)^{2}+\left(f_{2}-d_{1}\right)^{2}}\right]$


but $\sqrt{\left(e_{1}-a_{2}\right)^{2}+\left(f_{1}-b_{2}\right)^{2}}$ is the distance between the complex numbers $x=a+b i$ and $z=e+f i$, $\sqrt{\left(c_{1}-a_{2}\right)^{2}+\left(d_{1}-b_{2}\right)^{2}}$ is the distance between the complex numbers $x=a+b i$ and $y=c+d i$, and $\sqrt{\left(e_{1}-c_{2}\right)^{2}+\left(e_{2}-c_{1}\right)^{2}}$ is the distance between the complex numbers $y=c+d i$ and $z=e+f i$. Because the distance between the complex numbers is a metric, then: $d_{c}(x, y)+d_{c}(y, z) \leq d_{c}(x, z)$ and therefore $\sqrt{\left(c_{1}-a_{2}\right)^{2}+\left(d_{1}-b_{2}\right)^{2}}+\sqrt{\left(e_{1}-c_{2}\right)^{2}+\left(e_{2}-c_{1}\right)^{2}} \geq$ $\sqrt{\left(e_{1}-a_{2}\right)^{2}+\left(f_{1}-b_{2}\right)^{2}}$. Similarly, we can prove that: $\sqrt{\left(c_{2}-a_{1}\right)^{2}+\left(d_{2}-b_{1}\right)^{2}}+\sqrt{\left(f_{1}-d_{2}\right)^{2}+\left(f_{2}-d_{1}\right)^{2}}$ $\geq$ $d I(X, Y)+d I(Y, Z)$

$=\left[\sqrt{\left(c_{1}-a_{2}\right)^{2}+\left(d_{1}-b_{2}\right)^{2}}+\right.$

$\sqrt{\left(e_{1}-c_{2}\right)^{2}+\left(e_{2}-c_{1}\right)^{2}}$;

$\left.\sqrt{\left(c_{2}-a_{1}\right)^{2}+\left(d_{2}-b_{1}\right)^{2}}+\sqrt{\left(f_{1}-d_{2}\right)^{2}+\left(f_{2}-d_{1}\right)^{2}}\right]$

$\geq\left[\sqrt{\left(e_{1}-a_{2}\right)^{2}+\left(f_{1}-b_{2}\right)^{2}} ; \sqrt{\left(e_{2}-a_{1}\right)^{2}+\left(f_{2}-b_{1}\right)^{2}}\right.$

$=d I(X, Z)$.

Although $d I$ is not a metric, in fact the range of $d I$ is not $\mathbb{R}$ and is a partial function, this satisfies, as seen in propositions 3.4, 3.6 and 3.7, weak properties of metrics. Thus it is reasonable to consider $d I$ as a kind of "distance" between interval complex numbers.

\section{Definition 18 (A partial interval metric):}

Let the set $A$. A partial function $d_{p i}: A \times A \rightarrow \mathbb{R}$ is a partial interval metric if it satisfies the following properties $\forall X, Y, Z \in A$
a. Partial symmetry: If $d_{p i}(X, Y)$ is defined, then $d_{p i}(X, Y)=d_{p i}(Y, X)$
b. Sub-reflexivity: $[0,0] \subseteq d_{p i}(X, X)$
c. Partial triangle inequality: if $d_{p i}(X, Y), d_{p i}(Y, Z)$ and $d_{p i}(X, Z)$ are defined, then: $d_{p i}(X, Z) \leq_{K M}$ $d_{p i}(X, Y)+d_{p i}(Y, Z)$

Thus, by propositions $3.4,3.6$ and $3.7, d I$ is a partial interval metric.

Proposition 3.8: If $x \in X, y \in Y$ and $X$ and $Y$ are comparable. Then $d_{c}(x, y) \in d I(X, Y)$

Proof: It is trivial, as seen in figure 7.

Definition 19 (Module of an interval complex number based on Moore's distance):

Let $X=A+B i \in \mathbb{I} \mathbb{C}$. The module of $X$, denoted by $|X|$ is the non-negative real number $d_{M C}(X, 0)$, that is the Moore's distance from $X$ to the point $[0,0]+[0,0] i$.

Example 2:
Let $X=[-3,4]+[2,3] i$. Then,

$$
\begin{aligned}
|X| & =d_{M C}(X,[0,0]+[0,0] \\
& =\operatorname{Max}(|-3-0|,|2-0|)+\operatorname{Max}(|4-0|,|3-0|) \\
& =3+4 \\
& =7
\end{aligned}
$$

Definition 20 (Diameter of an interval complex number): Let $X=A+B i \in \mathbb{I} \mathbb{C}$. The diameter of $X$, denoted by $\operatorname{diam}(X)$, is the non-negative real number

$$
\sqrt{\operatorname{diam}(A)^{2}+\operatorname{diam}(B)^{2}}
$$

Definition 21 (Intersection of interval complex numbers):

Let $X=A+B i$ and $Y=C+D i \in \mathbb{I} \mathbb{C}$. The intersection of $X$ with $Y$ is the interval.

$$
\mathrm{X} \cap \mathrm{Y}=(\mathrm{A} \cap \mathrm{C})+(\mathrm{B} \cap \mathrm{D}) \mathrm{i}
$$

Intersection between two complex number intervals will only exist if $A \cap C$ and $B \cap D^{3}$ exist.

Proposition 3.9 (Monotonicity): Let $X, Y, Z, W \in \mathbb{I} \mathbb{C}$. If $X \subseteq Z$ and $Y \subseteq W$, then $X \cap Y \subseteq Z \cap W$

Proof: The proof can be found in [4] and [16].

\section{Polar Representation for interval complex numbers}

We are going to build a review in [4]: "If we attempt to express the set of complex numbers represented by an interval complex number, $A$, in its polar form, we encounter certain difficulties. Although the set of complex numbers determined by the absolute value and the amplitude of $A$ contains all the complex number elements of the interval complex number $A$, it will in general also contain a great many others. The shaded portions of 8 represent additional complex numbers included in a polar representation of a complex interval number.

Fig. 8. Original figure of the article [4] - A problem with the attempt of polar representation of an interval complex number considered by Boche.

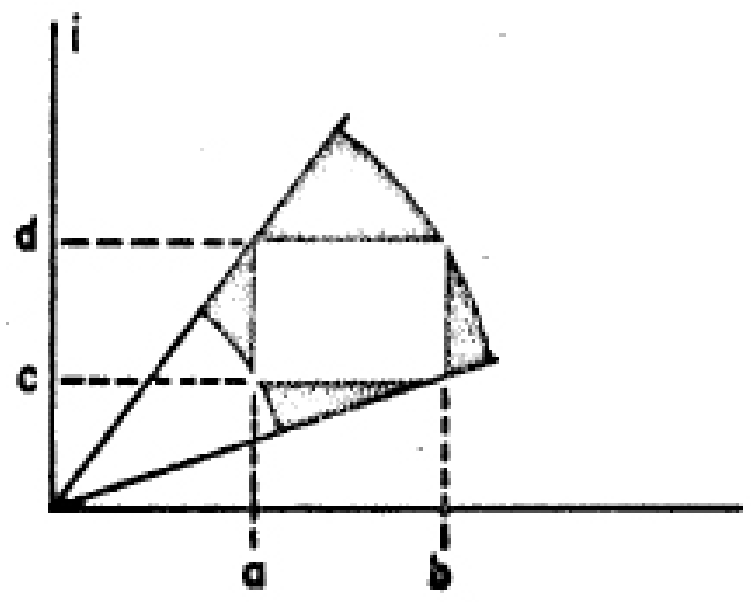

${ }^{3}[r, s] \cap[t, u]$ exist, if only if, $r \leq t \leq s$ or $t \leq r \leq u$. 
Such a polar representation could serve as the basis for an alternative definition of interval complex numbers. We could of course determine a rectangular region containing all elements of such a polar representation. However, it is easy to see that the only interval complex numbers for which the polar and Cartesian representations are equal have one of the three forms:

$$
\begin{aligned}
& {[a, a]+[b, b] i} \\
& {[c, d]+[0,0] i}
\end{aligned}
$$

or

$$
[0,0]+[c, d] i
$$

where 0 is not contained in the open interval $(c, d)$. Observe that in this text, written by Boche in [4], he does not define a polar representation for complex interval numbers. But, implicitly he considers the intervals $\left[\phi_{1}, \phi_{2}\right]$ and $\left[P_{1}, P_{2}\right]$ showed in figure 9 as the element of a "polar representation" of a complex interval number $A+B i$. However, he himself admitted that this representation is not adequate because there are complex numbers $a+b i$ so that the elements of their polar representation are $\phi$ and $P$, and $\phi \in\left[\phi_{1}, \phi_{2}\right]$ and $P \in\left[P_{1}, P_{2}\right]$, nevertheless $a \notin A$ or $b \notin B$.

Fig. 9. The attempt of polar representation of an interval complex number considered by Boche.

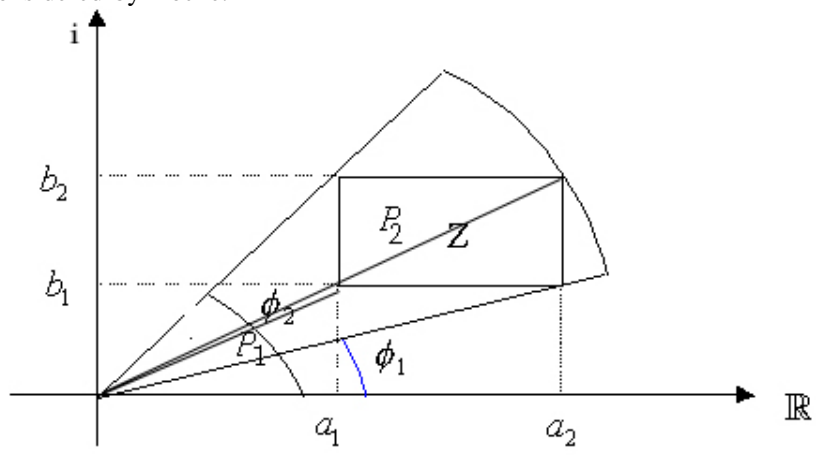

Still, that is not the greatest problem with this "polar representation" considered by Boche. The strongest problem is that the angles are not related with the amplitude. It, as we will see as follows, will not allow us to recover the cartesian representation of an interval complex number (in a different form of the three cases mentioned by him) from this polar representation, and conversely, it will not allow us to recover the elements of this polar representation from their cartesian representation. In other words, the Euler's identity will not be satisfied.

In our view, an interval complex number $\left[a_{1}, a_{2}\right]+\left[b_{1}, b_{2}\right] i$ is a closed rectangle, can also be seen as the interval of complex numbers $\left[a_{1}+b_{1} i, a_{2}+b_{2} i\right]$, the vertices of the rectangle are complex numbers $a_{1}+b_{1} i, a_{1}+b_{2} i, a_{2}+b_{1} i$ and $a_{2}+b_{2} i$. But, if we consider figure 9, as "polar representation" of an interval complex number $Z=A+B i=\left[a_{1}, a_{2}\right]+$ $\left[b_{1}, b_{2}\right] i$, and consider the Euler's identity, we will have:

$$
\begin{aligned}
Z & =P e^{\phi i} \\
& =\left[P_{1}, P_{2}\right] e^{\left[\phi_{1}, \phi_{2}\right] i} \\
& =\left[P_{1}, P_{2}\right]\left[e^{\phi_{1} i}, e^{\phi_{2} i}\right] \text { as } P_{1}, P_{2}, e^{\phi_{1}} \mathrm{e} e^{\phi_{2}} \text { are positive, } \\
& \text { then } \\
& =\left[P_{1} e^{\phi_{1} i}, P_{2} e^{\phi_{2} i}\right] \\
& \neq\left[a_{1}+b_{1} i, a_{2}+b_{2} i\right] \\
& =A+B i \text { there is no relation between } P_{i} \text { and } \phi_{i}
\end{aligned}
$$

Thus, we do not reconstruct the cartesian form from the polar form. To solve this problem, we need to obtain angles which relate with the amplitudes. We have two natural possibilities: the first is to consider the angles $\phi_{1}$ and $\phi_{2}$ in figure 9 with the amplitudes $P_{1}^{\prime}=\sqrt{a_{2}^{2}+b_{1}^{2}}$ and $P_{2}^{\prime}=\sqrt{a_{1}^{2}+b_{2}^{2}}$. Clearly, $\phi_{1}$ and $P_{1}^{\prime}$ are the elements of the polar representation of the complex number $a_{2}+b_{1} i$, and $\phi_{2}$ and $P_{2}^{\prime}$ are the elements of the polar representation of the complex number $a_{1}+b_{2} i$. But, this "polar representation" has the following problems:

1) There exist complex numbers $a+b i$ whose polar coordinates are in the intervals $\left[\phi_{1}, \phi_{2}\right]$ (or in $\left[\phi_{2}, \phi_{1}\right]$ ) and $\left[P_{1}^{\prime}, P_{2}^{\prime}\right]$ (or in $\left[P_{2}^{\prime}, P_{1}^{\prime}\right]$ ), but $a \notin\left[a_{1}, a_{2}\right]$ or $b \notin\left[b_{1}, b_{2}\right]$.

2) There exist complex numbers $a+b i$ whose polar representation is not in the intervals $\left[\phi_{1}, \phi_{2}\right]$ (or in $\left[\phi_{2}, \phi_{1}\right]$ ) and $\left[P_{1}^{\prime}, P_{2}^{\prime}\right]$ (or in $\left[P_{2}^{\prime}, P_{1}^{\prime}\right]$ ), but $a \in\left[a_{1}, a_{2}\right]$ and $b \in\left[b_{1}, b_{2}\right]$.

3) It does not satisfy Euler's identity. Suppose that it satisfies Euler's identity, then $A+B i=$ $=P e^{\phi i}$

$=\left[P_{1}, P_{2}\right] e^{\left[\phi_{1}, \phi_{2}\right] i}$

$=\left[P_{1}, P_{2}\right]\left[e^{\phi_{1} i}, e^{\phi_{2} i}\right]$ as $P_{1}, P_{2}, e^{\phi_{1}} \mathrm{e} e^{\phi_{2}}$ are positive, then

$=\left[P_{1} e^{\phi_{1} i}, P_{2} e^{\phi_{2} i}\right]$

$=\left[a_{2}+b_{1} i, a_{1}+b_{2} i\right]$

$\neq A+B i$ there is no relation between $P_{i}$ and $\phi_{i}$

The second possibility is to consider $P_{1}$ and $P_{2}$ of figure 9 and the angles $\gamma_{1}$ and $\gamma_{2}$ showed in figure 10, where $P_{1}$ is the minimum module of the vertices of the closed rectangle, $P_{2}$ is the maximum module of the vertices of the closed rectangle, showed in figure 11 , and $\gamma_{1}$ and $\gamma_{2}$ are angles built between $P_{1}$ and $P_{2}$ whose axis, respectively, i.e. $P_{1}=\min \left\{\left|a_{1}+b_{1} i\right|,\left|a_{1}+b_{2} i\right|,\left|a_{2}+b_{1} i\right|,\left|a_{2}+b_{2} i\right|\right\}$ and $P_{2}=\max \left\{\left|a_{1}+b_{1} i\right|,\left|a_{1}+b_{2} i\right|,\left|a_{2}+b_{1} i\right|,\left|a_{2}+b_{2} i\right|\right\}$. In figures 10 and 11 , we consider the polar representations of the points $a_{1}+b_{1} i$ and $a_{2}+b_{2} i$ in both cases. Clearly, this polar representation will have the first of the problems pointed above and the second, but only with respect to the angle. Still, as we will prove below, this approach does not have the last of the these problems, which from our point view is the main of the three. By construction $a_{1}=P_{1} \cos \gamma_{1}$ and $a_{2}=P_{2} \cos \gamma_{2}$ where $\gamma_{1}, \gamma_{2} \in \phi$. This could be better visualized by comparison between figures 9 and 10:

On the other hand, $\gamma_{1}$ and $\gamma_{2}$ do not form an interval, which can be clearly seen in figure 11 which $\gamma_{1} \leq \gamma_{2}$ does not always happen. However, one can consider the following interval: $\gamma=\left[\min \left\{\gamma_{1}, \gamma_{2}\right\}, \max \left\{\gamma_{1}, \gamma_{2}\right\}\right]$ By the Euclidian metric: $P_{1}=\sqrt{a_{1}^{2}+b_{1}^{2}}$ e $P_{2}=\sqrt{a_{2}^{2}+b_{2}^{2}}$ it can also be seen that $P_{1} \cos \gamma_{1}=a_{1}, P_{2} \cos \gamma_{2}=a_{2}, P_{1} \operatorname{sen} \gamma_{1}=b_{1}$ e 


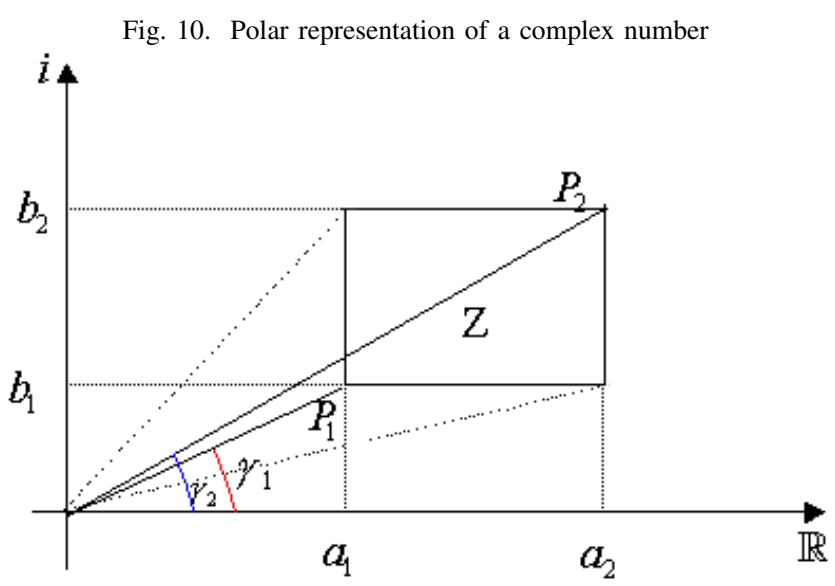

Fig. 11. Polar representation in which $P_{1}$ is in the third quadrant

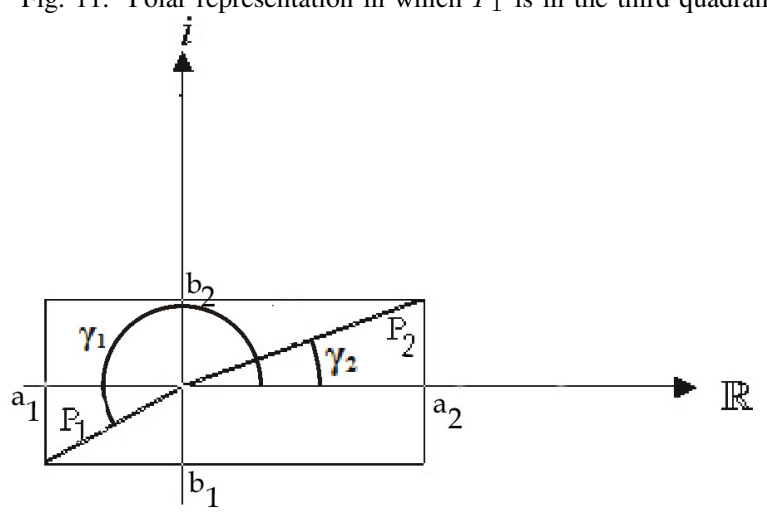

This way, the relation in the polar form in our point of view will be about the extremes and not in the interval. It also will not be about $\phi$, as it was thought previously, but about $\gamma_{1}$ and $\gamma_{2}$. Thus, Euler's Identity can be extended to the interval.

1) Euler's Interval Identity:

Theorem 3.11 (Euler's interval identity):

Let $Z \in \mathbb{I} \mathbb{C}$, where $Z=A+B i$ is the Cartesian representation and $Z=\left[P_{1} e^{\gamma_{1} i}, P_{2} e^{\gamma_{2} i}\right]$ is the polar representation, so:

e

$$
e^{\gamma_{1} i}=\cos \gamma_{1}+\sin \gamma_{1} i
$$

$$
e^{\gamma_{2} i}=\cos \gamma_{2}+\sin \gamma_{2} i
$$

$$
\begin{aligned}
& \text { Proof: } \\
& \begin{array}{l}
Z \quad=A+B i \\
\quad=\left[a_{1}, a_{2}\right]+\left[b_{1}, b_{2}\right] i \\
\quad=\left[a_{1}+b_{1} i, a_{2}+b_{2} i\right]
\end{array} \\
& \text { but } a_{1}+b_{1} i, a_{2}+b_{2} i \in \mathbb{C} \\
& \text { so } a_{1}+b_{1} i=P_{1} e^{\gamma_{1} i}=\cos \gamma_{1}+\sin \gamma_{1} i \\
& \text { and } a_{2}+b_{2} i=P_{2} e^{\gamma_{2} i}=\cos \gamma_{2}+\sin \gamma_{2} i \\
& \text { so }\left[e^{\gamma_{1} i}, e^{\gamma_{2} i}\right]=\left[\cos \gamma_{1}+\sin \gamma_{1} i, \cos \gamma_{2}+\sin \gamma_{2} i\right]
\end{aligned}
$$

This is trivial because, since the limits of the interval are complex numbers, thus they can be worked out as shown previously in the section "Review about Complex Numbers".

Example 3 (Euler's interval identity):

$P_{2} \operatorname{sen} \gamma_{2}=b_{2}$. so $\frac{\operatorname{sen} \gamma_{1}}{\cos \gamma_{1}} \frac{b_{1}}{a_{1}}=\tan \gamma_{1}$ then $\gamma_{1}=\arctan \left(\frac{b_{1}}{a_{1}}\right)$ in the same way $\frac{\operatorname{sen} \gamma_{2}}{\cos \gamma_{2}} \frac{b_{2}}{a_{2}}=$ $\tan \gamma_{2}$ then $\gamma_{2}=\arctan \left(\frac{b_{2}}{a_{2}}\right)$.

Theorem 3.10 (Polar representation of an interval complex number):

Let $Z \in \mathbb{I} \mathbb{C}$ and the polar coordinates $P_{1}, P_{2}$, module of $Z$ and $\gamma_{1}, \gamma_{2}$ the polar angle, then the polar representation of $Z$ is:

$$
Z=\left[P_{1} e^{\gamma_{1} i}, P_{2} e^{\gamma_{2} i}\right]
$$

Proof:

Let $Z=A+B i$ where $A=\left[a_{1}, a_{2}\right]$ and $B=\left[b_{1}, b_{2}\right]$, then:

Making $\mathrm{Z}$ from Cartesian form to polar form:

$\Rightarrow Z=\left[a_{1}+a_{1}\right]+\left[b_{1}, b_{2}\right] i$

$\Rightarrow Z=\left[a_{1}+b_{1} i, a_{2}+b_{2} i\right]$

$\Rightarrow \quad Z=\left[P_{1} e^{\gamma_{1} i}, P_{2} e^{\gamma_{2} i}\right]$

Remaking $\mathrm{Z}$ from polar form to Cartesian form:

$\Rightarrow Z=\left[P_{1} e^{\gamma_{1} i}, P_{2} e^{\gamma_{2} i}\right]$

$\Rightarrow \quad Z=\left[P_{1} \cos \left(\gamma_{1}\right)+i P_{1} \operatorname{sen}\left(\gamma_{1}\right), P_{2} \cos \left(\gamma_{2}\right)+i P_{2} \operatorname{sen}\left(\gamma_{2}\right)\right]$

$\Rightarrow Z=\left[P_{1} \cos \left(\gamma_{1}\right), P_{2} \cos \left(\gamma_{2}\right)\right]+\left[P_{1} \operatorname{sen}\left(\gamma_{1}\right), P_{2} \operatorname{sen}\left(\gamma_{2}\right)\right] i$

$\Rightarrow \quad Z=A+B i$

$$
\begin{aligned}
P_{2} & =\sqrt{5^{2}+3^{2}} \\
& =\sqrt{34} \\
& =5.830952
\end{aligned}
$$

$$
\begin{aligned}
\gamma_{1}^{\prime} & =\arctan \left(\frac{2}{2}\right) \\
& =0.785398
\end{aligned}
$$$$
\begin{aligned}
\gamma_{2}^{\prime} & =\arctan \left(\frac{3}{5}\right) \\
& =0.5404195
\end{aligned}
$$

$$
\begin{aligned}
Z & =\left[P_{1} e^{\gamma_{1} i}, P_{2} e^{\gamma_{2} i}\right] \\
& =\left[2.828427 e^{0.5404195 i}, 5.830952 e^{0.785398 i}\right] \\
& =[2.828427,5.830952] e^{[0.5404195,0.785398] i}
\end{aligned}
$$

Remaking $Z$ in Cartesian form:

$$
\begin{aligned}
= & {[2.828427 \cos (0.785398), 5.830952 \cos (0.5404195)]+} \\
= & {[2.828427 \operatorname{sen}(0.785398), 5.830952 \operatorname{sen}(0.5404195)] i } \\
= & {[2,5]+[2,3] i }
\end{aligned}
$$

So, we recovered exactly the cartesian representation of $Z$ from their polar representation, which is an excellent result. On the other hand, one needed to analyze what happens when the calculation is carried out using the interval $\gamma$ :

Let $\quad P \quad=$
$\gamma=[0.5404195,0.785398]$.

[2.828427, 5.830952] and 
Then $Z=[2.828427,5.830952] e^{[0.5404195,0.785398] i}$

$$
\begin{aligned}
P \cos \gamma & =[2.828427,5.830952] \cos [0.5404195,0.785398] \\
& =[2.828427,5.830952][0.7071069,0.8574929] \\
& =[2.000000,5.000000] \\
& =A \\
P \sin \gamma & =[2.828427,5.830952] \sin [0.5404195,0.785398] \\
& =[2.828427,5.830952][0.5144958,0.7071067] \\
& =[1.4552137,4.1231050] \\
& \supseteq B
\end{aligned}
$$

In this case, the cartesian representation obtained from the polar representation of $Z$ is exactly the same for $A$ but not for $B$, nevertheless the value obtained for $B$ approximate $B$, in the sense that it contains $B$. This occurred because, at present, the interval $\gamma$ is being used instead of the extremities as earlier and thus resulted in obtaining better results.

Example 4:

Let the complex interval $Z=[-2,2]+[-2,3] i$

$$
\begin{array}{rlrl}
P_{1} & =\sqrt{(-2)^{2}+(-2)^{2}} & P_{2} & =\sqrt{(2)^{2}+3^{2}} \\
& =\sqrt{8} & & =\sqrt{13} \\
& =2.828427 & & =3.605551 \\
\gamma_{1}^{\prime} & =\pi+\arctan \left(\frac{-2}{-2}\right) & & \\
& =\pi+0.785398 & & =\arctan \left(\frac{3}{2}\right) \\
& =3.926991 & & =0.982794 \\
Z & =\left[P_{1} e^{\gamma_{1} i}, P_{2} e^{\gamma_{2} i}\right] & \\
& =\left[2.828427 e^{0.982794 i}, 3.605551 e^{3.926991 i}\right] \\
& =[2.828427,3.605551] e^{[0.982794,3.926991] i}
\end{array}
$$

Remake $Z$ in Cartesian form:

$$
\begin{aligned}
Z= & {[2.828427 \cos (3.926991), 3.605551 \cos (0.982794)]+} \\
& {[2.828427 \operatorname{sen}(3.926991), 3.605551 \operatorname{sen}(0.982794)] i } \\
= & {[-2,2]+[-2,3] i }
\end{aligned}
$$

Thus, excellent results were obtained. On the other hand, we

need to analyze what happens if we calculate using the interval $\gamma$ : let $P=[2.828427,3.605551], \gamma=[0.982794,3.926991]$

then $Z=[2.828427,3.605551] e^{[0.982794,3.926991] i}$

$$
\begin{aligned}
P \cos \gamma & =[2.828427,3.605551] \cos [0.982794,3.926991] \\
& =[2.828427,3.605551][-0.707106,0.554700] \\
& =[-2.549507,2.000000] \\
& \supseteq A \\
P \sin \gamma & =[2.828427,3.605551] \sin [0.982794,3.926991] \\
& =[2.828427,5.830952][-0.707106,1] \\
& =[-4.123106,5.830952] \\
& \supseteq B
\end{aligned}
$$

Again, as can be seen, this result is good, but it is not perfect

because the values found for $A$ and $B$ are not exact, but it approaches $A$ and $B$.

\section{CONCLUSION}

In the present work, important definitions were introduced (definitions and theorems 9, 10, 3.1, 13, 16, 17, 18, 19 and 3.11) to the theory of complex variables and complex interval variables by defining basic complex elements under the knowledge of interval in defining a polar representation and presenting Euler's identity for the interval case. Using these concepts was important to the field of pure mathematics where one studies the mathematical interval, as well as in several other applications in the fields of science and engineering where the information about complex numbers is used and those can be treated as under numerical intervals, such as computational geometry, fazorial analysis, digital processing of images and signals, etc. These new aspects of intervals associated with practical problems, which use complex mathematics, until now being worked on punctual forms will offer support to the solutions presented and they will treat the solution within a clear margin as well [1]. We believe that the present work contributed to the enhancement of mathematical knowledge, which deals with the Interval Theory.

\section{REFERENCES}

[1] Benedito Melo Acióly, Fundamentação computacional da matemática intervalar, Ph.D. thesis, Instituto de Informática, Universidade Federal do Rio Grande do Sul, 1991.

[2] Hans-Robert Arndt, On interval systems $[x]=[a][x]+[b]$ and the powers of interval matrices in complex interval arithmetics, Reliable computing (2007), no. 13, 245-259.

[3] Frithjof Blomquist, Werner Hofschuster, Walter Krämer, and Markus Neher, Complex interval functions in c-xsc, Preprint 2005/2Wissenschaftliches Rechnen/ Softwaretechnologie, 2005.

[4] Ray Boche, Complex interval arithmetic with some applications, Lockheed Missiles \& Space Company- Sunnyvale, California (1966), 1-33.

[5] Yves Candau, Tarek Raissi, Nacim Ramdani, and Laurent Ibos, Complex interval arithmetic using polar form, Reliable Computing 12 (2006), no. 1, 1-20.

[6] Gonzales, Rafael and Wintz, P., Digital Image Processing, Second ed., Addison and Wesley Publishing, 1987.

[7] Irwin, J. David, Basic Engineering Circuit Analysis, Third ed., Macmillan Publishing, 1992.

[8] Luc Jaulin, Path planning using intervals and graphs, Reliable Computing - Kluwer Academic Publishers 7 (2001), 1-15.

[9] Michel Kieffer, Luc Jaulin, and Éric Walter, Robust autonomous robot localization using interval analysis, Reliable Computing 6 (2000), no. 6, 337-362, Kluwer Academic Publishers. Printed in the Netherlands.

[10] Kulisch, U.K and Miranker, W.L, Computer Arithmetic Theory and Practice, Academic Press, 1981.

[11] Olivier Leveque, Luc Jaulin, Dominique Meizel, and Eric Walter, Vehicle localization from inaccurate telemetric data: a set inversion approach, 5th IFAC Symposium on Robot Control SY.RO.CO.'97,.

[12] Mascarenhas, N.D.A and Velasco, F.R.D, Processamento Digital de Imagens, IV Escola de Computação 1 (1985), 235.

[13] Ramon. E. Moore, Interval analysis, Prentice Hall, New Jersey (1966).

[14] Ramon E. Moore, Methods and applications of interval analysis, Studies in Applied Mathematics - SIAM, Philadelfia, 1979.

[15] Moore, Ramon E., Computational functional analysis , Ellis Horwood Limited, Chichester, 1985.

[16] Paulo Werlang Oliveira, Tiaraj Asmuz Diverio, and Dalcidio Moraes Cláudio, Fundamentos da matemática intervalar, Sagra Luzzato, 1997.

[17] Rocha, Luis M. and Kreinovich, Vladik, Computing Uncertainty in Interval Based Sets. In Applications of Interval Computer, Kluwer Academic Press, 1996.

[18] Fabiana T Santana, Uma fundamentação para sinais e sistemas intervalares, Ph.D. thesis, Universidade Federal do Rio Grande do Norte UFRN, Dezembro 2011.

[19] Scott, Dana, Outline of a Mathematical Theory of Computation, Master's thesis, Oxford University Computing Laboratory, Okford, 1970. 
[20] Spiegel, Murray Ralph, Complex variables: resum of the theory, First ed., McGraw-Hill, 1981.

[21] Toussaint, Godfried T., Computational Morphology : A Computational Geometric Approach to the Analysis of Form, Six ed., Software Books, 2000. 